\title{
Multiple coding strategies in the retention of musical tones by possessors of absolute pitch
}

\author{
ROBERT J. ZATORRE \\ Montreal Neurological Institute and Hospital, McGill University, Montreal, Canada \\ and \\ CHRISTINE BECKETT \\ McGill University, Montreal, Canada
}

\begin{abstract}
Eighteen musicians with absolute pitch (AP) confirmed by screening tests participated in tonal and verbal short-term-retention tasks. In the tonal task, subjects identified three successive piano tones by their letter names. Recall of these note names after $18 \mathrm{sec}$ of counting backwards was near perfect. Recall after an 18-sec delay filled with random piano tones was also near perfect. In contrast, the same subjects demonstrated significant forgetting when required to retain letter trigrams while counting backwards for 18 sec. These results were essentially replicated in a second experiment using longer $(27 \mathrm{sec})$ retention intervals, a more demanding verbal interference task, and an active musical interference task (singing a descending scale). We interpret these results as indicating that retention of note names by possessors of AP is not limited to verbal encoding; rather, multiple codes (e.g., auditory, kinesthetic, and visual imagery) are probably used.
\end{abstract}

People who can identify by note name the pitch of a large number of musical tones, and who can accurately produce the pitch of a note without making use of a reference tone, are said to possess absolute pitch (AP). Considerable research has been carried out with these relatively rare individuals, because they seem to be an exception to Miller's (1956) formulation about the limitation of absolute identification along a stimulus continuum (for a review of AP see Ward \& Burns, 1982).

Several investigators have tried to determine the mechanisms used by possessors of AP to account for their performance on pitch-identification or -production tasks. For example, it seems clear that AP possessors do not simply rely on a superior pitch-discrimination ability (Oakes, 1955; Siegel, 1972) as has been suggested by some authors (e.g., Neu, 1947). Another hypothesis that has been suggested is that possessors of AP are able to encode the stimulus verbally and then use the verbal label for subsequent processing. In an important study, Siegel

\footnotetext{
We wish to thank the subjects who participated in this study for their time and cooperation. We also are grateful to the McGill University Faculty of Music, and particularly to the following persons: John Fisher, Joel Wapnick, Kathleen Toomey, Paul Pedersen, and D. Bruce Minorgan. This research was supported in part by Operating Grant MA 9598 from the Medical Research Council of Canada to the first author. Very helpful suggestions regarding Experiment 2 were made by A. Samuel and G. Lockhead. C. Beckett is a member of the Faculty of Music at McGill University. Correspondence may be addressed to Robert J. Zatorre, Neuropsychology Department, Montreal Neurological Institute and Hospital, 3801 University St., Montreal, Quebec H3A 2B4, Canada.
}

(1974) found that subjects who had AP could retain pitch information for sinusoidal signals over a filled interval of $15 \mathrm{sec}$, but only when the pitch separation was one semitone. When the separation was $1 / 10$ of a semitone, their forgetting rate over time was equivalent to that of a control group without AP. Siegel interpreted these results as evidence for verbal encoding on the part of the AP possessors, which allowed them to retain the information in the condition where the tones mapped onto the musical scale. When the verbal label could not be applied, all subjects had to rely on a sensory-coding strategy, thus producing a rapid decay (see also Bachem, 1954).

Although Siegel's (1974) results are consistent with a verbal-coding strategy, her data do not require such an interpretation. A musician's ability to use AP information when the pitch of the stimulus matches an internalized standard could very well depend on nonverbal processes. In particular, there is no direct evidence that a verbal label (as opposed to some other code) was being used to retain the information in the delay interval in Siegel's (1974) task.

One straightforward way to examine this issue is to take advantage of the well-known interference effects that exist in short-term memory (STM) tasks. Brown (1958) and Peterson and Peterson (1959) demonstrated that retention of verbal information (letter trigrams) in STM depends upon rehearsal; when rehearsal is blocked by an interfering task, such as counting backwards, rapid forgetting occurs. Retention of pitch information also shows rapid decay from STM for non-AP subjects (Massaro, 1970; Wicklegren, 1966), and the effect of interfering tonal 
material is highly disruptive (Deutsch, 1970; Zatorre, 1983). Of greatest relevance to the question at hand is Deutsch's (1970) finding that verbal interference appears to have no effect on the retention of pitch information. She demonstrated that subjects who did not possess AP could discriminate the pitch of two tones just as well when a series of spoken digits was interpolated between the two as when only silence was presented in the delay interval. This contrasts with the significantly disruptive effect of tonal interference material. Thus, the STM system for pitch in non-AP subjects seems to be at least in some ways distinct from that for verbal information.

Given the foregoing findings, we may postulate that if Siegel's hypothesis is correct (i.e., if AP possessors encode and retain pitch information solely or primarily by means of a verbal label), then this verbal encoding should be subject to the type of verbal interference effects described by Peterson and Peterson (1959). On the other hand, if such individuals are able to make use of one or more nonverbal codes, as Deutsch's results might suggest, then perhaps they will not demonstrate verbal interference effects but instead might be subject to interference from interpolated tonal material.

\section{EXPERIMENT 1}

We hypothesized that AP possessors, although quite able to give a verbal label in response to a particular pitch, are not limited to using solely a verbal code. To provide a strong test of this hypothesis, we prepared a task that seemed likely to induce the greatest possible verbal encoding on the part of AP possessors (naming a series of notes). We then required them to retain these verbal labels over time intervals filled with verbal or tonal interference. Our prediction was that possessors of AP would be able to retain pitch information even when verbal interference was present, which would contradict Siegel's (1974) proposal. As a control task, the same subjects retained verbal information (letter trigrams) over filled intervals.

\footnotetext{
Method

Subjects

Twenty-one musicians served as subjects. Two subjects were excluded because of difficulties with experimental equipment during their testing sessions. One other subject was excluded for failing to complete all tests. The remaining 18 subjects ranged in age from 13 to 50 years, with a mean of 32.4 years. They had spent an average of 16 years studying their principal instruments. No subject reported a history of hearing loss or neurological impairment. All subjects stated that they were possessors of absolute pitch. Fifteen subjects were right-handed and three reported mixed handedness.

\section{Stimulus Materials}

Musical stimuli were played on a 9-ft Mason and Hamlin grand piano in a large rehearsal hall and were recorded using a Toshiba RT-SF5 cassette machine and a Shotgun cylindrical microphone on Type I Normal Position suspended $30 \mathrm{~cm}$ above the strings.

The tasks fell into two categories, AP screening and pitch STM. Four identification tests were prepared for screening, and two further tests were prepared for evaluating pitch STM function.
}

AP screening tests. In the first two conditions, notes ranged upwards from "Great" C (approximately $65 \mathrm{~Hz}$ ) to $\mathrm{g}^{3}$ (approximately $1568 \mathrm{~Hz}$ ). In Identification Test 1 , the notes were chosen from a set of 12 separated by perfect fourths from $\mathrm{C}$ to $\mathrm{g}^{3}$ (Ward \& Burns, 1982). No note was duplicated across octaves (i.e., all chromas were different). The resulting 12 notes were presented as a set four times in random order, for a total of 48 notes. Identification Test 2 used the same range but included only Cs and Gs. This set of 9 notes was tested four times in random order, for a total of 36 notes.

Identification Test 3 restricted the range to one octave $c^{1}-c^{2}$ (approximately 262 to $523 \mathrm{~Hz}$ ). These 13 notes were presented four times in random order; in the last randomization, $\mathrm{c}^{2}$ was missing. This test thus held 51 notes. Identification Test 4 tested rising melodic intervals in the range $c^{1}-a^{2}$ (approximately 262 to $880 \mathrm{~Hz}$ ). Twelve intervals ranging from the minor second to the perfect octave were tested, the set being presented twice in random order, for a total of 48 notes.

In the first three conditions, both the intensity and the length of notes were varied in a nonsystematic fashion in an attempt to avoid revealing any clues provided by the piano itself. To allow the subjects time to respond, a gap of approximately $10 \mathrm{sec}$ was left between individual notes and a gap of $5 \mathrm{sec}$ was left between intervals. Intervals were played at a rate of 50 M.M., slightly slower than one note per second, and at a moderate dynamic level (i.e., mezzo forte)

Pitch STM tests. Test 1 consisted of 16 three-note groups on the natural notes in the octave between $c^{1}$ and $c^{2}$. Each group consisted of three different tones presented at a rate of about one note per second and at a moderate dynamic level. Rising, falling, V, and inverted $V$ contours were presented twice each in random order. Each group was followed by two-alternative forced-choice foils counterbalanced for true and false, in which the second tone in the set of three was altered by one scale step for the false examples. Interference for Pitch STM Test 1 was verbal (see below).

Test 2 used the same three-note groups as did Pitch STM Test 1, but each group was presented in reverse order. Foils were also reversed. Rate and intensity of presentation were as in the first test. Musical interference was recorded between stimuli and foils at the rate of two notes per second. The interference notes were chosen from the chromatic scale from $c^{1}$ to $c^{2}$ and were presented in random order.

Verbal STM Test 1 consisted of 24 consonant trigrams, or threeletter combinations, randomly chosen from the consonants of the alphabet (no vowels). These materials were taken from Corsi (1972).

Verbal STM Test 2 also consisted of 24 three-letter combinations, but using only the last seven letters of the alphabet $(T, U, V, W$, $\mathrm{X}, \mathrm{Y}, \mathrm{Z}$ ) in order to parallel the memory demands to the musical names.

\section{Procedure}

The subjects were tested individually in a quiet room. Free-field playback was on a Hitachi D-85s cassette deck, through a Lenco 900 turntable-amplifier and KLH 26 speakers. The identification screening test was presented first, in the order of Condition 1 through Condition 4 . The subjects notated the stimuli on music manuscript paper and named the intervals in Condition 4 by their short theoretical symbols (M3, P5, m7, etc).

The STM tasks then were tested in this order: Verbal STM Test 1 (full alphabet, verbal interference), Pitch STM Test 1 (verbal interference), Verbal STM Test 2 (restricted alphabet, verbal interference), and Pitch STM Test 2 (musical interference).

In Verbal STM Test 1 , the subjects were told to listen carefully as a three-letter combination was read to them slowly and evenly, about one letter per second; they were then to repeat the combination immediately. Next, they heard a three-digit number; they were to repeat the number and count backwards from it by ones. Each subject was allowed to count at his/her own rate. They were told 
Table 1

Pitch Identification Accuracy: Percent Correct Chroma Identification and Mean Absolute Semitone Deviations for Three Identification Tests

\begin{tabular}{|c|c|c|c|c|c|c|c|c|}
\hline \multirow[b]{2}{*}{ Subject } & \multicolumn{2}{|c|}{ TEST 1} & \multicolumn{2}{|c|}{ TEST 2} & \multicolumn{2}{|c|}{ TEST 3} & \multicolumn{2}{|c|}{ Mean } \\
\hline & $\%$ Correct & $M$ Dev. & $\%$ Correct & $M$ Dev. & $\%$ Correct & $M$ Dev. & $\%$ Correct & $M$ Dev. \\
\hline W.S. & 31.3 & 1.0 & 97.2 & 0.1 & 37.3 & 0.8 & 55.3 & 0.7 \\
\hline C.M. & 47.9 & 0.5 & 0.0 & 1.0 & 60.8 & 0.4 & 36.2 & 0.6 \\
\hline M.D. & 97.9 & 0.0 & 97.2 & 0.1 & 100.0 & 0.0 & 98.4 & 0.0 \\
\hline A.C. & 79.2 & 0.3 & 100.0 & 0.0 & 96.1 & 0.0 & 91.8 & 0.1 \\
\hline B.D. & 97.9 & 0.0 & 100.0 & 0.0 & 100.0 & 0.0 & 99.3 & 0.0 \\
\hline R.M. & 97.9 & 0.0 & 100.0 & 0.0 & 100.0 & 0.0 & 99.3 & 0.0 \\
\hline C.L. & 43.8 & 1.2 & 94.4 & 0.3 & 80.4 & 0.5 & 72.9 & 0.7 \\
\hline S.B. & 100.0 & 0.0 & 100.0 & 0.0 & 100.0 & 0.0 & 100.0 & 0.0 \\
\hline C.A. & 93.8 & 0.1 & 69.4 & 0.3 & 90.2 & 0.1 & 84.5 & 0.2 \\
\hline R.A. & 97.9 & 0.0 & 100.0 & 0.0 & 98.0 & 0.0 & 98.6 & 0.0 \\
\hline D.M. & 43.8 & 1.3 & 97.2 & 0.1 & 98.0 & 0.0 & 79.7 & 0.5 \\
\hline G.G. & 45.8 & 0.9 & 97.2 & 0.1 & 84.3 & 0.2 & 75.8 & 0.4 \\
\hline G.A. & 62.5 & 1.1 & 66.6 & 0.6 & 98.0 & 0.0 & 75.7 & 0.6 \\
\hline C.R. & 27.1 & 1.7 & 94.4 & 0.3 & 72.5 & 0.2 & 64.7 & 0.8 \\
\hline R.B. & 100.0 & 0.0 & 100.0 & 0.0 & 98.0 & 0.0 & 99.3 & 0.0 \\
\hline B.M. & 100.0 & 0.0 & 100.0 & 0.0 & 100.0 & 0.0 & 100.0 & 0.0 \\
\hline A.N. & 85.4 & 0.2 & 100.0 & 0.0 & 100.0 & 0.0 & 95.1 & 0.1 \\
\hline L.D. & 93.8 & 0.1 & 100.0 & 0.0 & 98.0 & 0.0 & 97.3 & 0.0 \\
\hline Mean & 74.8 & 0.5 & 89.6 & 0.2 & 89.5 & 0.1 & 84.7 & 0.3 \\
\hline
\end{tabular}

that it was important to count accurately and that they would be asked to remember the letter combination at the end of the counting. They were told that the length of time they were asked to count would vary. If the subject could not remember the letter group, he/she could think for a few moments and give whatever recall he/she had; each subject was allowed two chances for recall and was encouraged to guess if necessary. The verbal interference task was carried out for delays of $3,6,9,12,15$, and $18 \mathrm{sec}$ from the time the experimenter finished saying the number. The signal for recall was the experimenter's asking, "And the letters were ...?" The order of the different interference durations was varied at random in the 24-item test (but the durations were the same for all subjects). The subjects performed two practice items (not included in the main test items) with interference of 3 and $18 \mathrm{sec}$. Verbal STM Test 2 was carried out in identical fashion to Verbal STM Test 1 .

During Pitch STM Test 1, the subjects listened to three-note melodic groups and immediately named them. Either solfege (do, re, mi) or English (C, D, E) note-name systems were accepted. After identification, the subjects then counted backwards from a given three-digit number as in Verbal STM Test 1, with the exception that delays were of 9 and $18 \mathrm{sec}$ only. The subjects then renamed the notes from memory; two chances were given as in Verbal Test 1 . If the subject did not recall the three note names, foils were presented; the subject was told to listen to both complete foils before indicating his/her choice. Two practice examples with interference of 9 and $18 \mathrm{sec}$, respectively, were given to all subjects before starting the main 16 -item test.

In Pitch STM Test 2, the subjects again heard and named threenote groups. Interference consisted of the taped musical sequences of random chromatic tones for 9 or $18 \mathrm{sec}$, after which the subjects named the three stimulus notes again; they were allowed two chances as in all other tests. In cases of faulty recall, foils consisted of either hearing notes on the tape or having three-note groups named verbally. If the first eight foil sets were verbal, the remaining eight were musical; the subjects were randomized for foil order. In both music tests, foils that were not needed because of correct recall were not presented.

\section{Results}

The results of the pitch-identification screening tests are presented in Table 1, which shows the percent correct tone-chroma identification ${ }^{1}$ and the mean absolute deviation in semitones. As expected, Test 1 elicited the lowest overall performance; however, the error rates for Tests 2 and 3 were almost identical. Results for Test 4 (interval identification) are not presented because all subjects performed perfectly on this task. As shown in the table, there was a relatively wide range of performance among subjects who claimed AP ability. In some cases, a low score does not necessarily reflect poor AP. For example, Subject C.M. received $0 \%$ correct on Test 2 by transposing the entire test one semitone down, whereas other subjects made less systematic types of errors on at least some of the tests. The subjects with poorer AP ability were retained in the study so that the relationship between AP accuracy and short-term-retention ability could be examined.

The principal results of the STM tasks are shown in Figure $1 .^{2}$ The typical decay over time is evident for the two verbal tasks for both the full- and the restrictedalphabet conditions. In the restricted-alphabet condition, a Wilcoxon signed-rank test ${ }^{3}$ between the 0 - and 18-sec delays yielded a significant value $(W=159, p<.001)$. The average decay at $18 \mathrm{sec}$ was $24.1 \%$. The same comparison between the 0 - and 18 -sec delay also was significant for the full-alphabet condition $(W=129, p<.005$ ); the average decay was $15.9 \%$. Although the full-alphabet condition appeared somewhat more difficult overall, at $18 \mathrm{sec}$ this trend reversed so that the score was significantly lower for the restricted-alphabet condition ( $W=$ $169, \mathrm{p}<.001)$ than it was for the full-alphabet condition. 


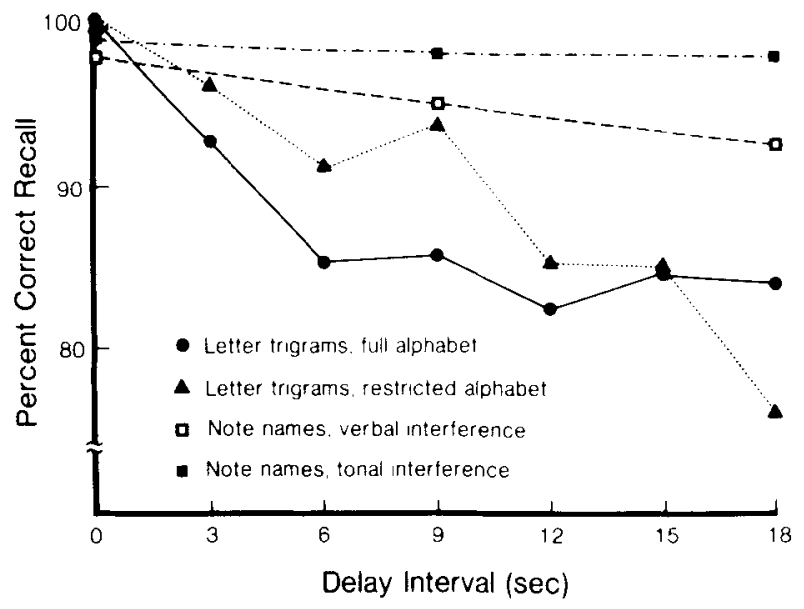

Figure 1. Mean percent correct recall for letter trigrams and note names with verbal and tonal interference as a function of delay interval for Experiment 1. The standard error of the mean was low in all cases, but tended to increase slightly with increasing delay. For verbal tasks, it varied from 1.7 to 4.0. For pitch tests, it varied from 1.9 to 2.2 .

For the pitch tasks, retention of the three-note stimuli showed little or no decay over the 18 -sec retention interval. At both 9- and 18-sec delays, for example, 8 subjects performed perfectly in Pitch Test 1 . If we examine the change between 0 and $18 \mathrm{sec}$ for Pitch Test 1 , there was only a marginally significant decay of performance, using a Wilcoxon test $(W=74, p=.055)$. Only 2 subjects scored below the $80 \%$ level at the 18 -sec delay, and the average decrease was only $5.3 \%$. For Pitch Test 2 , with tonal interference, there was no evidence for any loss of information; 14 of the 18 subjects scored perfectly in the 18-sec-delay condition.

On the occasions when recall of pitch information was not entirely correct, a two-alternative forced choice was given between the correct alternative and a foil. In Pitch Test 1 , this was required 13 times at the 9-sec delay and 21 times at the 18-sec delay. Recognition of the correct alternative under these circumstances was generally high: $92 \%$ at $9 \mathrm{sec}$ and $95 \%$ at $18 \mathrm{sec}$. In Pitch Test 2, foils were presented only on four occasions at each delay, because performance was generally perfect. Recognition in all cases when foils were presented was $100 \%$.

Comparison of each subject's performance at the 18sec delay between the verbal tests and Pitch Test 1 demonstrated that recall of letters was significantly poorer than was recall of notes (with the full-alphabet test, $W=55$, $p<.05$; with the restricted-alphabet test, $W=112$, $p<.002)$. In the restricted-alphabet condition, 4 subjects scored equally well as they did in Pitch Test 1 at the 18-sec delay, and only 2 were better with letters; the other 12 subjects performed worse with letters than they did with notes (overall difference between the two conditions was $14.4 \%$ ).

To assess the possible relationship between pitchidentification accuracy and short-term-retention ability, a Pearson product-moment correlation coefficient was calculated between average percent correct chroma-identification scores and percent correct recall at $18 \mathrm{sec}$ for Pitch Test 1 . The obtained value of 0.47 is significant ( $p=.05$ ), suggesting that the subjects who identified the tones well also retained them well. However, this result may be largely attributable to the fact that 1 subject (C.R.) was the poorest of all subjects on both tests; if we eliminate this subject's data and recalculate the correlation, it falls to .40 , which is not significant $(p>.10)$. No significant relationship was observed across subjects between retention of note names in Pitch Test 1 and retention of letter trigrams in either Verbal Test 1 or 2 (correlation coefficients were -0.27 and -0.28 , respectively). ${ }^{4}$

\section{Discussion}

The results of Experiment 1 clearly demonstrate that verbal interference has little or no effect on the short-term retention of note names by individuals who possess AP. The same subjects demonstrated a significant drop in recall of three spoken letters after $18 \mathrm{sec}$ of verbal interference, even when an alphabet restricted to seven letters was used, in accordance with the well-established effects originally described by Brown (1958) and Peterson and Peterson (1959). These results contradict Siegel's (1974) hypothesis that AP possessors differ from nonpossessors only in that the former have available to them a verbal label that can be rehearsed. If this had been the case, a decay of notename information over the 18 -sec verbal interference period would have been observed to the same degree as that observed with the letter trigrams.

One difficulty in comparing the note-retention task with the letter task is that there are only seven note names available in the scale used. For this reason, the restrictedalphabet condition was implemented. One might predict that such a condition would be easier than the full-alphabet condition, insofar as the probability of a correct answer by chance is increased. On the other hand, the possibility of a greater buildup of proactive interference may partially counteract this effect. In fact, the results indicate that both alphabet conditions resulted in significant loss of information over time, with a somewhat greater loss at $18 \mathrm{sec}$ for the restricted-alphabet condition. Thus, the excellent retention of note names observed in both pitch tests evidently was not related to the fact that the note names were drawn from a restricted set of seven.

\section{EXPERIMENT 2}

Despite the clear dissociation obtained in Experiment 1, a number of outstanding questions remain. In particular, the two tasks (letter vs. note retention) were not directly comparable because different numbers of trials and different delay intervals were used. For example, the larger number of trials in the verbal task might have led to a greater buildup of proactive interference (see Keppel \& Underwood, 1962). Another potential problem is that the 
delay intervals might not have been long enough to allow an interference effect to appear in the musical task. Also, in Experiment 1, the subjects were asked to count backwards by ones for comparability with results of other studies; this might not have provided sufficient interference in some cases. Finally, there is the objection that the musical interference task of Experiment 1 is not comparable to the verbal interference task, insofar as the former is entirely passive, requiring simply that the subjects listen, whereas the latter is active, requiring the subjects to produce numbers.

The purpose of Experiment 2 was to examine these questions: we prepared tonal and verbal tasks similar to those of Experiment 1, but with exactly comparable task parameters, longer retention intervals, and a more difficult verbal distracting task of counting backwards by threes in time to a metronome. In addition, to further our understanding of interference effects, we included an active musical interference task (singing a descending scale), along with the verbal interference task.

\section{Method}

\section{Subjects}

A group of 8 musicians served as subjects in Experiment 2. Five subjects had participated in Experiment 1, and 3 were newcomers. At least 16 months had elapsed between Experiments 1 and 2. The subjects' ages ranged from 23 to 49 years, with a mean of 33 years. They had spent an average of 15 years studying their principal instruments. No subject reported a history of hearing loss or neurological impairment. All subjects stated that they were possessors of AP. Seven of the 8 subjects were right-handed.

\section{Stimulus Materials}

Stimuli were recorded under conditions identical to those of Experiment 1.

Pitch STM test. Test 1 consisted of 12 three-note groups on the natural notes in the octave between $\mathrm{c}^{1}$ and $\mathrm{c}^{2}$. Each group consisted of three different tones presented at a rate of about one note per second and at a moderate dynamic level. The stimuli were identical to 12 of the 16 groups used in Experiment 1. Test 2 used the same three-note groups as Pitch STM Test 1 , but each group was presented in reverse order; rate and intensity of presentation were as in the first test.

Verbal STM tests. Both Verbal Tests 1 and 2 consisted of 12 consonant trigrams, using only the last seven letters of the alphabet in order to parallel the memory demands of the musical names. Verbal Test 2 used the trigrams of Test 1 in reverse order.

Interference. Verbal interference consisted of counting backwards by threes from a given three-digit number at a rate of 40 per minute. For musical interference, subjects hummed or sang a descending chromatic scale from a given note at the rate of 40 notes per minute. For each subject, all 12 chromatic notes were given in turn, in random order, as the starting note for the musical interference task. The pace was set and maintained throughout both interference tasks by a Franz LM-FB-4 electric metronome set at 40 M.M. Interference duration periods were 9,18 , and $27 \mathrm{sec}$, with each duration being presented in a random order four times within each test/task combination.

\section{Procedure}

The subjects were tested individually in a quiet room. Free-field playback was from a Hitachi D-E2 cassette deck through a Marantz 1060 amplifier and EPI M-100 speakers.

The order of presentation of tests and the combination of tests with interference tasks were counterbalanced across subjects in such a way that half of the subjects received the pitch tests first followed by the verbal tests; within each subgroup, the order of interference task also was balanced, such that half of the subjects received verbal interference followed by musical interference, and vice versa.

In the verbal STM tests, the subjects were told to listen carefully as a three-letter combination was read to them slowly and evenly, about one letter per second; they were to repeat the combination immediately, at a similar pace. They then heard either a hummed musical tone or a three-digit number.

For verbal interference, the subjects were to repeat the number and count backwards from it by threes in time to the metronome. The metronome was turned on to coincide precisely with the subject's repetition of the given number. The subjects were told that it was important to count accurately and that they would be asked to remember the letter combination at the end of the counting. The signal for recall was the metronome's being turned off and the question "And the letters were ...?" They were told that the length of time that they would be asked to count would vary. Each subject was allowed only one chance for recall but was encouraged to say whatever he/she could, even if it was only a single letter from the original combination. The subject performed two practice items (not included in main test items), one with an interference of $9 \mathrm{sec}$ and another with an interference of $27 \mathrm{sec}$.

For musical interference, the subject hummed the given tone and then hummed down the chromatic scale (descending in semitones) in time to the metronome, which had again been started to coincide exactly with the first tone. Humming prevented overt note naming. The subjects were told it was important to sing in tune. The signal for recall was the metronome's being turned off and the question "And the notes were ...?"' In all respects (the varying lengths of interference, the sole chance at recall, and two practice items of the shortest and longest interference durations), the procedure for musical interference was identical to that for verbal interference.

During the pitch STM tests, the subjects listened to three-note groups and immediately named them. Either solfege (e.g., do, re, mi) or English (e.g., C, D, E) note-name systems were accepted. After identification, the subjects performed the verbal or musical interference tasks as described above. The subjects then renamed the notes from memory; one chance was given, as in the verbal tests. Two practice examples, with interferences of 9 and $27 \mathrm{sec}$, were given to all subjects before starting each main pitch STM test.

\section{Results}

The data from Experiment 2 are graphed in Figure 2, which clearly shows that only one condition, recall of letters with verbal interference, yielded significant forgetting over time. This is borne out by statistical comparisons between the 0 - and 27 -sec-delay conditions for each task. For note-name recall with humming as the interfering task, there was almost no interference effect: 6 of the 8 subjects performed perfectly at the longest delay. For note-name recall with verbal interference, there also was no evidence of any drop over time: 5 of the 8 subjects scored perfectly at $27 \mathrm{sec}$, and the difference between performance at 0 and $27 \mathrm{sec}$ was not significant $(W=6$, $p>.10$ ).

For recall of letter trigrams, there was absolutely no interfering effect from humming: all 8 subjects scored perfectly at the longest delay under this condition. The only significant forgetting occurred for recall of letters with verbal interference: all subjects showed some drop over $27 \mathrm{sec}$-the average decrease was $18.2 \%$, which is statistically significant $(W=30, p<.02)$. 


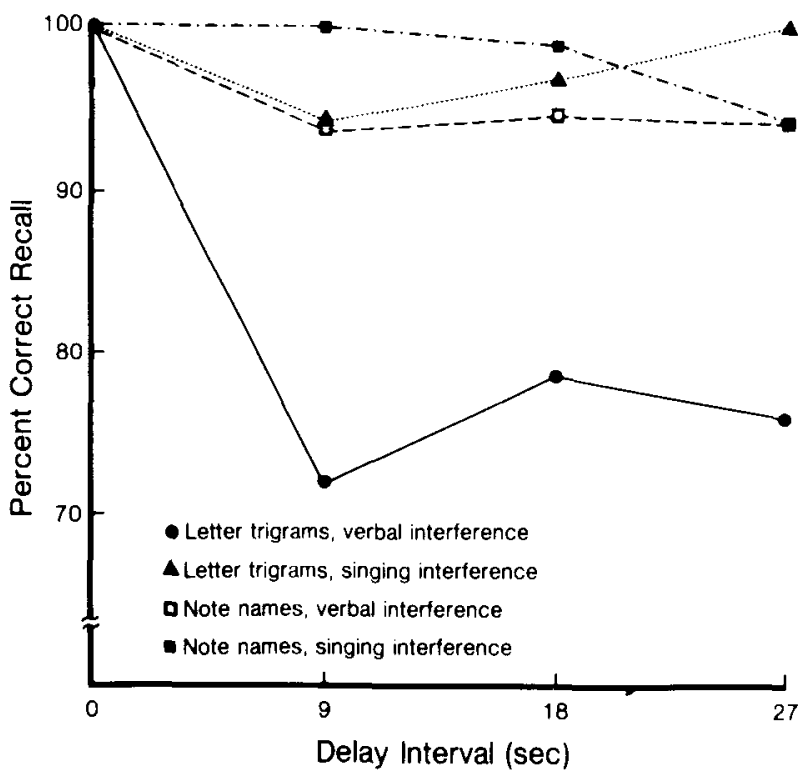

Figure 2. Mean percent correct recall for letter trigrams and note names with verbal and singing interference as a function of delay interval for Experiment 2. The standard error of the mean varied from 1.4 to 6.2 for verbal retention tasks and from 1.0 to 5.8 for tonal retention tasks, and tended to increase slightly with increasing delay.

The results of Experiment 2 may be compared directly to those of Experiment 1 at the 9- and 18-sec delays for the 5 subjects who participated in both experiments. Performance was very similar for this subsample between the two experiments. At the 9-sec delay, no significant differences between performance on Experiment 1 $(74.1 \%)$ and Experiment $2(75 \%)$ were noted $(t=.10)$. At the 18-sec delay, there also was essentially no difference between Experiment $1(75.8 \%)$ and Experiment 2 $(77.5 \% ; t=.08)$.

\section{Discussion}

The results of Experiment 2 replicate and extend our findings from Experiment 1. Retention of note names is not affected by verbal interference, even for as long as $27 \mathrm{sec}$. In comparison with Experiment 1, in which there was a marginally significant forgetting of note names with verbal interference, it is notable that there was no evidence for any forgetting under the (presumably more demanding) verbal interference condition of Experiment 2. Moreover, the active musical interference task, which is much more comparable to the verbal interference task, also failed to provide any interfering effect on retention of note names (or letter names).

Under the conditions of Experiment 2, verbal retention with verbal interference was clearly the most difficult condition. The drop over time, expected from previous studies using this type of paradigm, was apparent from the immediate repetition to the 9-sec delay, but there was no further forgetting from 9 to $27 \mathrm{sec}$. It is not clear why there was no further drop over time; perhaps there were not sufficient trials to bring out the difference between the three delay conditions. It is also somewhat curious that performance in this condition of Experiment 2 was comparable to verbal recall obtained in Experiment 1 , even though the interfering task was presumably more difficult. The subjects did experience a strong interference effect from counting backwards by threes, but this was no worse than what they had demonstrated in Experiment 1 . It is most unlikely that any practice effects can account for this, because well over 1 year had elapsed between the two experiments.

In any case, the same dissociation as in Experiment 1 was observed between retention of three note names (which was excellent) and three letters (which was relatively poor) under verbal interference. Thus, the data from Experiment 1 cannot be explained away by incomparable task parameters, by the brevity of the retention interval, or by the ease of the interfering tasks.

\section{GENERAL DISCUSSION}

Our results support the notion that AP possessors have available to them a number of encoding strategies that may be used concurrently, including (but not limited to) verbal labeling. According to this view, AP possessors differ from nonpossessors in that they are able to match pitches of tones to some fixed internal scale, which in turn allows them to give the corresponding verbal label; however, they presumably can also effect the match without recourse to the label. Thus, if access to the verbal label is difficult or impossible, they should still be able to make use of their knowledge about the pitch of the tone. On the other hand, when the tones do not match an internalized template, AP possessors should have no advantage over nonpossessors, as was demonstrated by Siegel (1974) in the condition in which adjacent tones in the set were separated by only $1 / 10$ of a semitone. This account of AP is not meant to explain what factors may lead to its acquisition or development; rather, it points out the potential richness of the AP possessor's cognitive framework for pitch.

It might be argued that the comparison between the letter and note sequences is inappropriate insofar as the note sequences may contain additional relational information (e.g., contour, intervals formed between notes, etc.) that could facilitate recall. In particular, it is possible that relative pitch cues might have played a role in the subjects' recall. However, the speed and ease with which the subjects produced the three note names argues against their use of relative pitch for these judgments (also, no subject indicated using relative pitch cues in debriefing; having AP, they report having no need to do so). Another important factor is that piano tones were used in the present study (whereas Siegel, 1974, used sinusoids). The extra cues available in piano tones (particularly the timbral cues) might have facilitated the task and aided our subjects to rely on more than just verbal encoding (see Ward \& Burns, 1982). While these factors are undoubtedly sig- 
nificant, the tonal tasks required only the retention of the verbal code for the notes; at the end of the delay interval, the subjects produced only the note names. The experimental conditions were set up to favor verbal encoding in order to provide the strongest test of the hypothesis in question. The fact that the subjects succeeded in recall of note names despite this argues against their need to rely solely on such verbal labels.

It also might be argued that many strategies of encoding (such as chunking) are possible for letter trigrams, which would facilitate the subject's recall (indeed, several subjects did report idiosyncratic ways of recalling the trigrams because of their similarity to certain meaningful abbreviations). Nevertheless, it is difficult to retain letters in the Brown-Peterson paradigm, as has been known for many years. Considering that the verbal interference task does not even use the same class of stimuli as the items to be remembered (numbers vs. letters), perhaps the question should be why the letters are so easily forgotten, rather than why the note names are retained. One might well wonder why letters are not as easily amenable to multiple encoding (e.g., visual imagery) in this type of experimental paradigm as tones appear to be.

The subjects who participated in this study all claimed to possess AP. As can be seen from Table 1, there was a fairly wide range of ability in performance of the screening tests across subjects in Experiment 1, although some performed much better on some tests than did others. All subjects were retained in order to examine the relationship between AP ability and STM retention of pitch information. The range and type of errors exhibited by our subject sample is typical of that observed in groups of people who claim AP (Bachem, 1937; Lockhead \& Byrd, 1981), particularly with respect to the occurrence of octave errors. Despite several subjects' difficulties with some of the screening tests, all were able to name the three notes essentially perfectly when they were first presented in the retention tasks. Moreover, although there was a significant correlation between pitch identification scores on the screening tests and retention of the three notes with verbal interference, this was primarily attributable to 1 subject who scored poorly on all conditions. Thus, we may safely conclude that even subjects who make some errors in identifying isolated pitches, and whose AP is therefore not perfect, are able to retain pitch information in a STM paradigm with little difficulty.

Given the almost complete lack of interference effect of counting on retention of note-name information over either the $18 \mathrm{sec}$ of Experiment 1 or the $27 \mathrm{sec}$ of Experiment 2 , the following question arises: What sort of encoding might the subjects have been using such that verbal interference (or humming) had no effect? The subjects' own introspections may provide some clues to this. Most reported being able to retain an auditory image of the tone during the interference task. One subject's comments exemplify this strategy: "The sound is always there and it gives me the names again; no need to use names to recreate the sound." Many subjects also reported strate- gies related to how the note sequences would be performed on their instruments, especially hand positions and finger tensions. Some subjects went so far as to feel different degrees of tension according to which finger had the first, second, and last notes. A few subjects also reported visual images of the notes on their instrument, usually a keyboard, but 1 subject reported visualizing the notes on the staff (interestingly, the one error he made consisted of a transposition of the notes to a different position on the musical staff, consistent with a visual strategy).

It is interesting to note that both musical interference tasks (passive listening and humming) had essentially no effect on retention of note names, in agreement with Siegel's (1974) findings. This result also supports the possibility that multiple encoding of the three-note stimulus may have taken place under these conditions, and that if interference affects retention of one code, others may still be available. It may be argued that in Experiment 1, the tonal interference task is passive, in that the subjects simply listen, whereas in the verbal condition, they must actively count backwards. Experiment 2, in which the musical interference task was more active, demonstrates that, even under those conditions, the subjects are able to retain the note name, again suggesting the use of multiple codes. As noted above, Deutsch (1970) has gathered evidence for the hypothesis that STM mechanisms for tonal information may be to some extent separate from verbal STM. The present results would also be in accord with this notion.

At this point, we are not yet in a position to propose a complete explanation of the mechanisms behind the presumed encoding strategies that led to the results of the present experiments. Nevertheless, some interesting neuropsychological findings may provide a clue to the underlying neural substrate involved. Several years ago, Corsi (1972; see also Milner, 1978) reported that subjects with surgical excision of the left temporal cortex and hippocampal region demonstrated accelerated forgetting of letter trigrams in a Brown-Peterson type of task. Patients with left temporal cortical removals in whom the hippocampal region was intact, or those with excision involving tissue from the right temporal lobe, showed a normal rate of forgetting with the same task. Recently, we studied an individual possessor of AP who underwent left temporal lobectomy, including partial hippocampal excision, for relief of epileptic seizures (Zatorre, in press). This subject demonstrated a deficit in retention of letter trigrams with verbal interference, as expected. Of greatest interest was that his retention of note names under conditions similar to those of Experiment 1 was nearly flawless. We may speculate, on the basis of this result, that there is at least a partial degree of independence in the STM mechanisms responsible for retention of verbal as opposed to tonal materials. The former may be linked to the function of structures in the left temporal lobe, the latter, perhaps, to the right temporal lobe. The hypothesis that the neural substrate for these types of stimuli differs would be consistent with the results of the present 
study, as well as with the results of studies with non-AP subjects showing minimal interference effects of verbal retention on tonal discrimination (Deutsch, 1970).

It will be of interest, in future studies, to attempt to manipulate subjects' encoding strategies directly by means of instructions, or to vary the nature of the interfering task more, in order to add further support for the hypothesis that multiple encoding of tonal information can take place. Sinusoidal tones also should be tested to rule out the possibility that, with more impoverished signals, verbal encoding may take on greater importance.

\section{REFERENCES}

BАCHEM, A. (1937). Various types of absolute pitch. Journal of the Acoustical Society of America, 9, 146-151.

BACHEM, A. (1954). Time factors and absolute pitch determination. Journal of the Acoustical Society of America, 26, 751-753.

Brown, J. (1958). Some tests of the decay theory of immediate memory. Quarterly Journal of Experimental Psychology, 10, 12-21.

Corsi, P. (1972). Human memory and the medial temporal region of the brain. Unpublished doctoral thesis, McGill University, Montreal.

Deutsch, D. (1970). Tones and numbers: Specificity of interference in short-term memory. Science, 168, 1604-1605.

KEPPEL, G., UNDERWOOD, B. J. (1962). Proactive inhibition in shortterm retention of single terms. Journal of Verbal Learning \& Verbal Behavior, 1, 153-161.

LOCKHEAD, G. R., BYRD, R. (1981). Practically perfect pitch. Journal of the Acoustical Society of America, 70, 387-389.

MASSARO, D. W. (1970). Retroactive interference in short-term recognition memory for pitch. Journal of Experimental Psychology, 83, 32-39.

MiLler, G. A. (1956). The magical number seven plus or minus two: Some limits on our capacity for processing information. Psychological Review, 63, 81-97.

MILNER, B. (1978). Clues to the cerebral organization of memory. In P. Buser \& A. Rougeul-Buser (Eds.), Cerebral correlates of conscious experience (INSERM Symposium No. 6, pp. 139-153). Amsterdam: Elsevier/North Holland.
NEU, D. M. (1947). A critical review of the literature on "absolute pitch." Psychological Bulletin, 44, 249-266.

OAKES, W. F. (1955). An experimental study of pitch naming and pitch discrimination reactions. Joumal of Genetic Psychology, 86, 237-259.

Peterson, L. R., \& Peterson, M. J. (1959). Short-term retention of individual verbal items. Joumal of Experimental Psychology, 58, 193-198.

SiEgel, J. A. (1972). The nature of absolute pitch. In I. E. Gordon (Ed.), Studies in the psychology of music (Vol. 8). Iowa City: University of Iowa Press.

SiEGEL, J. A. (1974). Sensory and verbal coding strategies in subjects with absolute pitch. Journal of Experimental Psychology, 103, 37-44.

WARD, W. D., BURns, E. M. (1982). Absolute pitch. In D. Deutsch (Ed.), The psychology of music (pp. 431-451). New York: Academic Press.

WICKELGREN, W. A. (1966). Consolidation and retroactive interference in short-term recognition memory for pitch. Journal of Experimental Psychology, 72, 250-259.

ZatorRe, R. J. (1983). Category-boundary effects and speeded sorting with a harmonic musical-interval continuum: Evidence for dual processing. Journal of Experimental Psychology: Human Perception \& Performance, 9, 739-752

ZATORRE, R. J. (in press). Intact absolute pitch ability after left temporal lobectomy. Cortex.

\section{NOTES}

1. Tone chroma refers to the position of the tone within the octave. In order to be able to measure the average semitone deviation around the target, and in accordance with the suggestion of $W$ ard and Burns (1982), responses that were one or more octaves away from the true value were scored by considering them to have occurred within the correct octave.

2. The data were scored taking both accuracy and order of report into account. Rescoring for accuracy alone yielded very similar results and will not be discussed further.

3. Nonparametric statistics were used because of the highly skewed nature of the obtained distributions.

4. Correlations for Pitch Test 2 were not run because performance was nearly flawless for most subjects

(Manuscript received May 18, 1987; revision accepted for publication April 24, 1989.) 